\title{
Sex Differences in the Association between Living Environmental Factors and Nutritional Status in Community-Dwelling Elderly Koreans
}

\author{
Dong Eun Kim ${ }^{1, \dagger}$, Hee-Sook Lim ${ }^{2,+}{ }^{\oplus}$, Hyejin Ahn ${ }^{1,3} \mathbb{C}^{\circ}$, Young Sun Kim ${ }^{3,4, *}$ and \\ Yoo Kyoung Park $1,3, *$ (D) \\ 1 Department of Medical Nutrition, Graduated School of East-West Medical Science, Kyung Hee University, \\ Yongin 17104, Korea; kim-doeu@hanmail.net (D.E.K.); hjahn@khu.ac.kr (H.A.) \\ 2 Department of Food Sciences and Nutrition, Yeonsung University, Anyang 14011, Korea; \\ limhs@yeonsung.ac.kr \\ 3 New Aging Center, Kyung Hee University, Yongin 17104, Korea \\ 4 Department of Gerontology, Graduate School of East-West Medical Science, Kyung Hee University, \\ Yongin 17104, Korea \\ * Correspondence: ysunkim@khu.ac.kr (Y.S.K.); ypark@khu.ac.kr (Y.K.P.) \\ + These authors contributed equally to this work as first author.
}

Received: 29 June 2020; Accepted: 15 August 2020; Published: 19 August 2020

\begin{abstract}
The association between nutritional status and living environment among 703 communitydwelling participants (268 men and 435 women) aged 65 years and older was assessed. In this cross-sectional survey study, living environmental factors, health-related factors, and nutrition (the Nutrition Quotient for the Elderly scores; NQ-E) were assessed. NQ-E scores were significantly higher in men than women, as were diversity and behavior factor scores (diversity: men, $50.2 \pm 16.1$; women, $44.1 \pm 17.5$; behavior: men, $59.3 \pm 16.9$; women, $54.1 \pm 16.6$ ). Participants living with a spouse and engaging in frequent sports activities had significantly higher adjusted odds ratio (OR) for having a high NQ-E than those who lived alone and engaged in restful activities and hobbies (men: adjusted OR for high NQ-E $=8.99$; 95\% confidence interval (CI): 1.35-59.56; women: adjusted OR for high NQ-E $=5.62 ; 95 \%$ CI: 2.36-13.38). We confirmed that women's nutritional status, unlike men's, was better when proper nutrition education was provided and when food security was guaranteed. For all participants, social activities and networks were important for maintaining good nutritional status and a healthy life. We suggest that different nutritional management strategies are needed for elderly people depending on sex.
\end{abstract}

Keywords: elderly; nutrition quotient for elderly (NQ-E); dietary behaviors; living environment; social activity

\section{Introduction}

A super-aged society, where more than $20 \%$ of the total population is aged 65 years and older, is in our near future and represents an emerging global challenge [1]. Korea is expected to have the highest percentage of elderly people in the world, with the population composition of those aged 65 or older calculated to reach $43.9 \%$ in 2060, up from $14.9 \%$ in 2019 [2]. The European Union is emphasizing promotion of a healthy "active aging" society and active lifestyles among older adults [3]. As elderly peoples' activities and social participation increase, the desire to pursue values and to improve quality of life rather than to simply hope for survival is growing [4]. Aging is defined as the collective series of physiological changes that occur in an organism over time, resulting in progressive deterioration of functioning, increased vulnerability to disease, and reduced viability $[5,6]$. Specifically, various factors 
such as poor appetite, restricted activity, reduced income, social isolation, and depression cause many changes in physiological functioning and health conditions [7]. In addition, medication, hospitalization, and social aspects of aging can also contribute to nutritional inadequacy [8]. A cornerstone for healthy aging is optimal nutritional status. Early identification of older adults who are at risk for insufficient caloric intake and nutrient adequacy, termed nutritional risk, is paramount to maintaining health, independence, quality of life, and longevity $[9,10]$. Most studies have reported sex-specific differences in the nutritional status and community integration of elderly individuals. Kiefer et al. [11] suggested that women have greater awareness and better knowledge of nutrition and nutrient intake than men. By contrast, women are reported to have more eating problems and greater difficulty managing diets [12]. These differences indicate that numerous factors such as education, marital status, household composition, socioeconomic status, social support, and geographic and environmental characteristics affect nutritional status and eating behaviors $[13,14]$. Korea's poverty ratio of the old is higher than that of Organization for Economic Cooperation and Development (OECD) countries, and Korean elderly women have lower education and economic level than men and are more likely to live alone $[15,16]$. Women have a higher rate of sarcopenia and lower nutritional status than men due to the higher proportion of women who lack exercise [17]. Food diversity in sarcopenic women was also reported to be lower than in the non-sarcopenia population [15]. This suggests that nutritional status and dietary behaviors can be influenced by psychological and sociocultural factors and that the effects may vary by sex. Therefore, there is a need for better understanding the aspects of older men and women's living environments that affect nutritional status, dietary behavior, and dietary practice.

This study examined the differences between health-related conditions and environmental factors according to sex in community-dwelling elderly Koreans and the social environmental factors affecting nutritional status.

\section{Materials and Methods}

\subsection{Study Subjects}

A cross-sectional survey study was conducted in elderly community-dwelling Koreans between July 2019 and December 2019. The inclusion criteria were as follows: Korean nationality, current residence in Korea, and $\geq 65$ years old with no visual disturbance or severe vision impairment. Those who were illiterate, unable to understand texts, or unable to communicate were excluded. Simple random sampling and regional sampling were used as a sampling technique considering the regional distribution of subjects. We determined the sample size to be 700 people, based on a $99 \%$ confidence interval with a margin of error of $5 \%$ in 850 million persons aged 65 years or over in Korea in 2019 [18,19]. Using a self-administered questionnaire, 794 questionnaires were collected, and 703 questionnaires were ultimately used; 91 were excluded due to missing information or refusal to participate. This study was approved by the Institutional Review Board of Kyung Hee University under the Helsinki Research Principles (approval number: KHSIRB-19-005).

\subsection{Assessing Nutritional Status}

Participants' nutritional status was assessed using the Nutrition Quotient for Elderly (NQ-E), an evaluation tool for measuring food behavior and dietary quality in people aged 65 years or older. The NQ-E was developed and validated by the Korean Nutrition Society in 2018 based on the literature and on data from the Korean National Health and Nutrition Examination Survey [20]. The NQ-E questionnaire consists of 19 items categorized into four factors: "dietary behavior" (6 items addressing difficulties in chewing foods, perception of one's health, depressive symptoms, handwashing practices before eating meals, hours of exercise, and efforts to have healthy eating habits); "balance" (4 items addressing intake frequencies of milk or dairy products, fruits, snacks, and water); "diversity" (6 items covering intake frequencies of eggs, fishes or shellfishes, and beans or bean products; frequency of eating alone; number of vegetable dishes at each meal, excluding kimchi; and meal frequency); 
and "moderation" ( 3 items covering intake frequency of sweets and baked products, sugar-added beverages, and instant noodles). The weighted scores for all four factors were added, yielding the total NQ score, which ranged from 0 to 100. The participants were divided into 3 groups categorized by the Korean Nutritional Society [20] according to the total NQ score (high: 63.5-100, medium: 51.9-63.4, and low: 0-51.8).

\subsection{Demographic Characteristics, Body Mass Index (BMI), and Health-Related Factors}

Demographic characteristics such as age, sex, employment status, education, and monthly income were recorded. Body mass index (BMI) was calculated based on height and weight. This study used weight categories from the World Health Organization (WHO) Asia-Pacific Perspective and the Korean Society for the Study of Obesity: underweight and normal, $\leq 22.99 \mathrm{~kg} / \mathrm{m}^{2}$; overweight, $23.0-24.99 \mathrm{~kg} / \mathrm{m}^{2}$; and obese and severely obese, $\geq 25.00 \mathrm{~kg} / \mathrm{m}^{2}$ [21]. The number of chronic diseases (hypertension, dyslipidemia, stroke, myocardial infarction, arthritis, osteoporosis, diabetes mellitus, cancers, and digestive disorders) and medications, activities of daily living (ADL), usual form of transportation (walking only, bus, subway, car or taxi, and so forth), social support (social care services, home delivery meal service, and free meal service), and nutrition counseling experiences were recorded as health-related factors.

\subsection{Living Environmental Factors Related to Food Intake}

Living environmental factors included social and food intake-related factors. Living status (alone, with spouse, and with children or children's families), social activities (resting and hobbies, cultural and artistic, sports, or other activities), frequency of social activity (rarely, regular, and trying to be active), and social relations (at least 3 times per week, 1-2 times per week, fewer than 3 times per month, and almost none) were investigated as social factors. Food security (secure, mildly secure, and moderately or severely insecure), ability to cook, and nutrition knowledge (low, medium, and high) were assessed as food intake-related factors. Food security was evaluated using a three-item measure of food insecurity during the last 1 year: (1) "Do you have enough money to buy food you need most of the time?"; (2) "In the past year, have you skipped one or more meals because you had no food in the house or because you thought that you might not have enough food soon?"; and (3) "In the past year, have you had to choose between buying food and paying bills or buying something else you needed?" [22]. The nutrition knowledge questionnaire consisted of 10 true-false questions referring to prior research [23]: proteins have the most caloric nutrients, vitamins are calorie nutrients, fats are a nutrient that strengthens bones, soybeans have a lot of protein, milk is the best source of calcium, iron deficiency causes anemia, egg yolks contain a lot of cholesterol, sufficient water intake prevents constipation, people with hypertension should avoid salty foods, and fiber is a caloric nutrient. Scores were considered to indicate high (8-10 points), medium (4-7 points), and low (0-3 points) nutrition knowledge depending on the number of correct answers.

\subsection{Statistical Analyses}

Subjects' demographic characteristics and dietary factors are presented as mean and standard deviation for continuous variables and as frequency and percentage (\%) for categorical variables. Before conducting comparative analysis between groups, we identified that the data had a normal distribution. Group comparisons used chi-square tests for qualitative variables and independent $t$-tests or one-way analysis of variance (ANOVA) for quantitative variables. Subsequently, multinomial logistic regression analyses were performed to evaluate factors affecting the NQ-E. All analyses were carried out using the Statistical Package for Social Sciences for Windows version 25.0 (SPSS, Inc., Chicago, IL, USA). Statistical significance was set at 0.05 based on a two-sided test. 


\section{Results}

\subsection{Demographic Characteristics}

Participants' demographic characteristics are summarized in Table 1. The mean age was $74.4 \pm 6.4$ years (men, $74.3 \pm 6.2$; women, $74.4 \pm 6.6$ years). The level of education most frequently achieved was college or higher for men $(36.9 \%)$ and elementary school graduation $(35.6 \%)$ for women $(p<0.001)$. The most common employment status for both men and women was unemployed (men, 79.9\%; women, 87.4\%; $p<0.01$ ), and the most frequent income level for both was $<500,000$ won (men, $24.3 \%$; women, 39.8\%; $p<0.001$ ).

\subsection{Comparision of $N Q-E$ Total and Component Scoress by Sex}

Subjects' NQ-E scores are shown in Table 2. The mean NQ-E score was $58.7 \pm 11.1$. The mean NQ-E was significantly higher for men than for women $(59.9 \pm 10.6$ vs. $58.1 \pm 11.4$, respectively; $p<0.05)$. Men's scores on the diversity and behavior factors of the NQ-E were significantly higher than women's (diversity: $50.2 \pm 16.1$ vs. $44.1 \pm 17.5$, respectively; $p<0.001$; behavior: $59.3 \pm 16.9$ vs. $54.1 \pm 16.6$, respectively; $p<0.001$ ). The balance and moderation factors did not differ by sex. When compared by age, as the age of men and women increase, the average NQ score was decreased. Men showed significant differences in NQ-E, diversity, and behavior, while women showed differences in NQ-E, balance, diversity, moderation, and behavior.

\subsection{Sex Differences in Health-Related and Living Environmental Factors According to NQ-E Level}

Sex differences in health-related and living environmental factors according to NQ-E are presented in Tables 3 and 4 . In both men and women, significant differences were found according to NQ-E level (high, medium, and low) in the number of diseases and medications, ADL, current living status, social relations and activities, transportation, experience of social support by government, food security, and nutrition knowledge.

Participants with low NQ-E had the highest number of diseases and medications (men, $p=0.003$; women, $p=0.001$ ). Men and women with high NQ-E scores had the highest rate of living with their spouse (men, 91 (91.0\%); women, $91(65.5 \%)$ ). Women with low NQ-E had the highest rate of living alone $(89(65.9 \%))$. Men and women with high NQ-E had the highest number of social relations (at least 3 times/week), engagement in sports activities, and frequency of social activities. In terms of food security among both men and women, those with high NQ-E scores had the highest food security and nutrition knowledge. Women with high NQ-E had the highest cooking ability and the most nutritional counseling experience. For men, these factors did not vary significantly according to NQ-E scores.

\subsection{Effects of Living Environment on NQ-E by Sex: Multinomial Logistic Regressions}

The effects of living environments on NQ-E levels according sex are shown in Tables 5 and 6. Men and women living with a spouse had a significantly higher adjusted odds ratio (OR) for high NQ-E levels than those who were living alone (men: adjusted OR for high NQ-E $=8.99 ; 95 \%$ confidence interval (CI): 1.35-59.56; women: adjusted OR for high NQ-E = 5.62; 95\%CI: 2.36-13.38). Elderly men and women who participated in sports activities had a significantly higher adjusted OR for medium or high NQ-E levels compared to those who engaged in sedentary and hobby activities (men: adjusted OR for medium NQ-E = 3.41; 95\%CI: 1.18-9.80; women: adjusted OR for high NQ-E = 12.07; 95\%CI: 3.91-37.20). Among women, those who had social interactions at least 3 times/week had a significantly higher adjusted OR of high NQ-E levels than those with fewer social interactions (adjusted OR for high NQ-E = 16.04; 95\%CI: 1.72-149.18). 
Table 1. General characteristics of the subjects by sex.

\begin{tabular}{|c|c|c|c|c|c|c|c|c|c|c|}
\hline & \multirow{2}{*}{$\begin{array}{c}\text { Total } \\
(n=703)\end{array}$} & \multicolumn{8}{|c|}{ Sex } & \multirow[b]{2}{*}{$p$-Value ${ }^{(3)}$} \\
\hline & & \multicolumn{3}{|c|}{ Men $(n=268)$} & \multicolumn{4}{|c|}{ Women $(n=435)$} & \multirow[b]{3}{*}{$p$-Value ${ }^{(2)}$} & \\
\hline Average age, mean \pm SD & $74.38 \pm 6.41$ & \multicolumn{3}{|c|}{$74.32 \pm 6.17$} & \multicolumn{4}{|c|}{$74.41 \pm 6.55$} & & 0.869 \\
\hline Age & & $\begin{array}{c}65-74 \\
(n=148)\end{array}$ & $\begin{array}{c}75-84 \\
(n=101)\end{array}$ & $\begin{array}{c}\geq 85 \\
(n=19)\end{array}$ & $p$-Value ${ }^{(2)}$ & $\begin{array}{c}65-74 \\
(n=233)\end{array}$ & $\begin{array}{c}75-84 \\
(n=164)\end{array}$ & $\begin{array}{c}\geq 85 \\
(n=38)\end{array}$ & & $p$-Value ${ }^{(4)}$ \\
\hline \multicolumn{11}{|l|}{ Education, $n(\%)$} \\
\hline Uneducated & $94(13.4)$ & $5(3.4)$ & $7(6.9)$ & $4(21.1)$ & \multirow{5}{*}{0.000} & $18(7.7)$ & $45(27.4)$ & $15(39.5)$ & \multirow{5}{*}{0.000} & \multirow{5}{*}{0.000} \\
\hline Elementary school & $198(28.2)$ & $12(8.1)$ & $25(24.8)$ & $6(31.6)$ & & $79(33.9)$ & $58(35.4)$ & $18(47.4)$ & & \\
\hline Middle school & $97(13.8)$ & $14(9.5)$ & $8(7.9)$ & $2(10.5)$ & & $53(22.7)$ & 20 (12.2) & $0(0.0)$ & & \\
\hline High school & $173(24.6)$ & $58(39.2)$ & $25(24.8)$ & $3(15.8)$ & & $51(21.9)$ & $32(19.5)$ & $4(10.5)$ & & \\
\hline College or Higher & $141(20.1)$ & $59(39.9)$ & $36(35.6)$ & $4(21.1)$ & & $32(13.7)$ & $9(5.5)$ & $1(2.6)$ & & \\
\hline \multicolumn{11}{|l|}{ Employment, $n(\%)$} \\
\hline Employed & $109(15.5)$ & $41(27.7)$ & $12(11.9)$ & $1(5.3)$ & \multirow{2}{*}{0.002} & $40(17.2)$ & $12(7.3)$ & $3(7.9)$ & \multirow{2}{*}{0.010} & \multirow{2}{*}{0.008} \\
\hline Unemployed & $594(84.5)$ & $107(72.3)$ & $89(88.1)$ & $18(94.7)$ & & $193(82.8)$ & $152(92.7)$ & $35(92.1)$ & & \\
\hline \multicolumn{11}{|l|}{ Monthly income, $n(\%){ }^{(1)}$} \\
\hline$<50$ & $238(33.9)$ & 19 (12.8) & $34(33.7)$ & $12(63.2)$ & \multirow{5}{*}{0.000} & $59(25.3)$ & $87(53.0)$ & $27(71.1)$ & \multirow{5}{*}{0.000} & \multirow{5}{*}{0.000} \\
\hline $50-99$ & $179(25.5)$ & $26(17.6)$ & $22(21.8)$ & 4 (21.1) & & $74(31.8)$ & $44(26.8)$ & $9(23.7)$ & & \\
\hline 100-199 & $111(15.8)$ & $38(25.7)$ & 19 (18.8) & $2(10.5)$ & & $36(15.5)$ & $15(9.1)$ & $1(2.6)$ & & \\
\hline $200-299$ & 77 (11.0) & 18 (12.2) & $13(12.9)$ & $1(5.3)$ & & $36(15.5)$ & $8(4.9)$ & $1(2.6)$ & & \\
\hline$\geq 300$ & $98(13.9)$ & $47(31.8)$ & $13(12.9)$ & $0(0.0)$ & & $28(12.0)$ & $10(6.1)$ & $0(0.0)$ & & \\
\hline
\end{tabular}

$\mathrm{SD}=$ standard deviation; ${ }^{(1)}$ units $=10,000$ won; ${ }^{(2)} p$-values were expressed between age using a chi-square test.; ${ }^{(3)} p$-values were expressed between sex using an independent $t$-test; and ${ }^{(4)} p$-values were expressed between sex a chi-square test or independent $t$-test. 
Table 2. The level and scores of the Nutrition Quotient for Elderly (NQ-E) by sex.

\begin{tabular}{|c|c|c|c|c|c|c|c|c|c|c|c|}
\hline \multirow{3}{*}{\multicolumn{2}{|c|}{ Age }} & \multirow{3}{*}{ Total } & \multicolumn{8}{|c|}{ Sex } & \multirow[b]{3}{*}{$p$-Value ${ }^{(4)}$} \\
\hline & & & \multirow{3}{*}{$\begin{array}{c}\begin{array}{c}65-74 \\
(n=148)\end{array} \\
62(41.9)\end{array}$} & \multicolumn{2}{|c|}{ Men $(n=268)$} & \multirow[b]{2}{*}{$p$-Value ${ }^{(3)}$} & \multicolumn{4}{|c|}{ Women $(n=435)$} & \\
\hline & & & & $\begin{array}{c}75-84 \\
(n=101)\end{array}$ & $\begin{array}{c}\geq 85 \\
(n=19)\end{array}$ & & $\begin{array}{c}65-74 \\
(n=233)\end{array}$ & $\begin{array}{c}75-84 \\
(n=164)\end{array}$ & $\begin{array}{c}\geq 85 \\
(n=38)\end{array}$ & $p$-Value ${ }^{(3)}$ & \\
\hline \multirow{3}{*}{ NQ-E } & Low $^{(1)}$ & $195(27.7)$ & & $36(35.6)$ & $2(10.5)$ & \multirow{3}{*}{0.000} & $89(38.2)$ & 45 (27.4) & $5(13.2)$ & \multirow{3}{*}{0.000} & \multirow{3}{*}{0.042} \\
\hline & Medium & $269(38.3)$ & $66(44.6)$ & $35(34.7)$ & $7(36.8)$ & & $98(42.1)$ & $52(31.7)$ & $11(29.0)$ & & \\
\hline & High & $239(34.0)$ & $20(13.5)$ & $30(29.7)$ & $10(52.6)$ & & $46(19.7)$ & $67(40.9)$ & $22(57.9)$ & & \\
\hline \multicolumn{2}{|c|}{ Average NQ-E score ${ }^{(2)}$, mean \pm SD } & $58.74 \pm 11.12$ & $61.98 \pm 9.77$ & $58.11 \pm 11.33$ & $52.65 \pm 8.57$ & 0.000 & $60.60 \pm 10.31$ & $55.90 \pm 12.29$ & $51.85 \pm 9.25$ & 0.000 & 0.037 \\
\hline \multirow{3}{*}{ Balance } & Low $^{(1)}$ & $234(33.3)$ & $41(27.7)$ & $29(28.7)$ & $2(10.5)$ & \multirow{3}{*}{0.428} & $77(33.0)$ & $40(24.4)$ & $5(13.2)$ & \multirow{3}{*}{0.000} & \multirow{3}{*}{0.425} \\
\hline & Medium & $275(39.1)$ & $59(39.8)$ & $32(31.7)$ & $8(42.1)$ & & $109(46.7)$ & $51(31.1)$ & $16(42.1)$ & & \\
\hline & High & $194(27.6)$ & $48(32.4)$ & $40(39.6)$ & $9(47.4)$ & & $47(20.2)$ & $73(44.5)$ & $17(44.9)$ & & \\
\hline \multicolumn{2}{|c|}{ Average balance score, mean \pm SD } & $39.29 \pm 23.27$ & $40.67 \pm 21.45$ & $36.88 \pm 23.63$ & $38.40 \pm 22.34$ & 0.062 & $44.33 \pm 22.61$ & $35.54 \pm 25.08$ & $30.93 \pm 19.88$ & 0.000 & 0.424 \\
\hline \multirow{3}{*}{ Diversity } & Low & $249(35.4)$ & $36(24.3)$ & 37 (36.6) & $3(15.8)$ & \multirow{3}{*}{0.001} & $67(28.8)$ & $24(14.6)$ & $3(7.9)$ & \multirow{3}{*}{0.000} & \multirow{3}{*}{0.000} \\
\hline & Medium & $284(40.4)$ & $87(58.8)$ & $35(34.7)$ & $6(31.6)$ & & $101(43.4)$ & $48(29.3)$ & $2(5.3)$ & & \\
\hline & High & $170(24.2)$ & $25(16.9)$ & $29(28.7)$ & $10(52.6)$ & & $65(27.9)$ & $92(56.1)$ & $28(73.7)$ & & \\
\hline \multicolumn{2}{|c|}{ Average diversity score, mean \pm SD } & $46.45 \pm 17.20$ & $51.82 \pm 12.84$ & $50.11 \pm 18.91$ & $38.66 \pm 18.13$ & 0.003 & $48.81 \pm 16.37$ & $39.85 \pm 17.71$ & $33.87 \pm 14.20$ & 0.000 & 0.000 \\
\hline \multirow{3}{*}{ Moderation } & Low & $114(16.2)$ & $55(37.2)$ & $45(44.6)$ & $9(47.4)$ & \multirow{3}{*}{0.119} & $104(44.6)$ & $95(57.9)$ & $23(60.5)$ & \multirow{3}{*}{0.055} & \multirow{3}{*}{0.028} \\
\hline & Medium & $258(36.7)$ & $70(47.3)$ & $32(31.7)$ & $8(42.1)$ & & $89(38.2)$ & $48(29.3)$ & $11(19.0)$ & & \\
\hline & High & $331(47.1)$ & $23(15.5)$ & $24(23.8)$ & $2(10.5)$ & & $40(17.2)$ & $21(12.8)$ & $4(10.5)$ & & \\
\hline Average moder & core, mean \pm SD & $82.55 \pm 16.06$ & $81.97 \pm 14.77$ & $79.24 \pm 19.44$ & $84.43 \pm 17.19$ & 0.308 & $81.63 \pm 16.16$ & $85.60 \pm 14.45$ & $85.18 \pm 15.01$ & 0.033 & 0.063 \\
\hline \multirow{3}{*}{ Behavior } & Low & $196(27.9)$ & $77(52.0)$ & 37 (36.6) & $2(10.5)$ & \multirow{3}{*}{0.000} & $86(36.9)$ & $33(20.1)$ & $5(13.2)$ & \multirow{3}{*}{0.000} & \multirow{3}{*}{0.000} \\
\hline & Medium & $267(38.0)$ & $50(33.7)$ & $33(32.7)$ & 7 (36.9) & & $96(41.2)$ & $71(43.3)$ & $10(16.3)$ & & \\
\hline & High & $240(34.1)$ & $21(14.2)$ & $31(30.7)$ & $10(52.6)$ & & $51(21.9)$ & $60(36.6)$ & $23(60.5)$ & & \\
\hline Average beha & ore, mean \pm SD & $56.09 \pm 16.89$ & $62.97 \pm 15.43$ & $56.49 \pm 17.50$ & $46.15 \pm 15.75$ & 0.000 & $58.23 \pm 15.56$ & $50.46 \pm 16.32$ & $44.47 \pm 16.73$ & 0.000 & 0.000 \\
\hline
\end{tabular}

$\mathrm{NQ}-\mathrm{E}=$ nutritional quotient for elderly; ${ }^{(1)}$ data are expressed as frequencies and percentages (\%); ${ }^{(2)}$ data are express as means \pm standard deviations (SD); ${ }^{(3)} p$-values were expressed between age using a chi-square test or independent $t$-test; and ${ }^{(4)} p$-values were expressed between sex a chi-square test or independent $t$-test. 
Table 3. Health-related factors of the subjects according to NQ-E level.

\begin{tabular}{|c|c|c|c|c|c|c|c|c|}
\hline & \multicolumn{8}{|c|}{ NQ-E levels $(n=703)$} \\
\hline & \multicolumn{3}{|c|}{ Men $(n=268)$} & \multirow[b]{2}{*}{$p$-Value } & \multicolumn{4}{|c|}{ Women $(n=435)$} \\
\hline & $\begin{array}{c}\text { Low } \\
(n=60)\end{array}$ & $\begin{array}{l}\text { Medium } \\
(n=108)\end{array}$ & $\begin{array}{c}\text { High } \\
(n=100)\end{array}$ & & $\begin{array}{c}\text { Low } \\
(n=135)\end{array}$ & $\begin{array}{l}\text { Medium } \\
(n=161)\end{array}$ & $\begin{array}{c}\text { High } \\
(n=139)\end{array}$ & $p$-Value \\
\hline \multicolumn{9}{|c|}{$\mathrm{BMI}^{(1)}, n(\%)$} \\
\hline$\leq$ Normal & $32(53.3)$ & 35 (32.4) & $25(25.0)$ & & $67(49.6)$ & 62 (38.5) & $64(46.0)$ & \\
\hline Overweight & $14(23.3)$ & $42(38.9)$ & $42(42.0)$ & 0.007 & $36(26.7)$ & $46(28.6)$ & $33(23.7)$ & 0.283 \\
\hline$\geq$ Obesity & $14(23.3)$ & $31(28.7)$ & $33(33.0)$ & 0.007 & $32(23.7)$ & $53(32.9)$ & $42(30.2)$ & 0.200 \\
\hline Average BMI, mean \pm SD & $22.98 \pm 2.83$ & $23.75 \pm 2.44$ & $24.07 \pm 2.34$ & 0.030 & $22.97 \pm 3.20$ & $24.00 \pm 3.20$ & $23.78 \pm 3.00$ & 0.015 \\
\hline \multicolumn{9}{|c|}{ Numbers of diseases, $n(\%)$} \\
\hline 0 & $9(15.0)$ & $17(15.7)$ & $22(22.0)$ & & $6(4.4)$ & $13(8.1)$ & $24(17.3)$ & \\
\hline $1-2$ & $31(51.7)$ & $76(70.4)$ & $66(66.0)$ & 0.005 & $60(44.4)$ & $73(45.3)$ & $70(50.4)$ & 0.001 \\
\hline 3 or more & $20(33.3)$ & $15(13.9)$ & $12(12.0)$ & & $69(51.1)$ & $75(46.6)$ & $45(32.4)$ & \\
\hline Ave. no. of disease, mean \pm SD & $1.97 \pm 1.40$ & $1.45 \pm 0.98$ & $1.33 \pm 1.13$ & 0.002 & $2.86 \pm 1.79$ & $2.54 \pm 1.58$ & $1.96 \pm 1.52$ & 0.000 \\
\hline \multicolumn{9}{|c|}{ Number of medications, $n(\%)$} \\
\hline 0 & $11(18.3)$ & $22(20.4)$ & $29(29.0)$ & & $19(14.1)$ & $18(11.2)$ & $33(23.7)$ & \\
\hline $1-2$ & $35(58.3)$ & 77 (71.3) & $64(64.0)$ & 0.008 & $59(43.7)$ & $99(61.5)$ & $71(51.1)$ & 0.001 \\
\hline 3 or more & $14(23.3)$ & $9(8.3)$ & $7(7.0)$ & & $57(42.2)$ & $44(27.3)$ & $35(25.2)$ & \\
\hline Ave. no. of medication, mean $\pm S D$ & $1.72 \pm 1.31$ & $1.25 \pm 0.87$ & $1.14 \pm 1.04$ & 0.003 & $2.36 \pm 1.71$ & $2.06 \pm 1.40$ & $1.67 \pm 1.45$ & 0.001 \\
\hline \multicolumn{9}{|c|}{ Activities of daily living (ADL) } \\
\hline Requires no assistance & $32(53.3)$ & $85(78.7)$ & $92(92.0)$ & & $66(48.9)$ & 109 (67.7) & $112(80.6)$ & \\
\hline Some assistance needed & $28(46.7)$ & $23(21.3)$ & $8(8.0)$ & 0.000 & $69(51.1)$ & $52(32.3)$ & $27(19.4)$ & 0.000 \\
\hline \multicolumn{9}{|c|}{ Transportation ${ }^{(2)}, n(\%)$} \\
\hline Walking only & $29(48.3)$ & 20 (18.5) & $5(5.0)$ & & $60(44.4)$ & $63(39.1)$ & $15(10.8)$ & \\
\hline Bus & $30(50.0)$ & $51(47.2)$ & $29(29.0)$ & & $69(51.1)$ & $88(54.7)$ & $63(45.3)$ & \\
\hline Subway & $12(20.0)$ & $37(34.3)$ & $44(44.0)$ & 0.000 & $29(21.5)$ & $58(36.0)$ & $47(33.8)$ & 0.000 \\
\hline Car or taxi & $4(6.7)$ & $35(32.4)$ & $37(37.0)$ & & $20(14.8)$ & $13(8.1)$ & $36(25.9)$ & \\
\hline Etc. ${ }^{(3)}$ & $2(3.3)$ & $5(4.6)$ & $7(7.0)$ & & $3(2.2)$ & $4(2.5)$ & $1(0.7)$ & \\
\hline \multicolumn{9}{|c|}{ Experience of social support by government ${ }^{(4)}, n(\%)$} \\
\hline Yes & $8(13.3)$ & $12(11.1)$ & $2(2.0)$ & & $39(28.9)$ & $21(13.0)$ & $11(7.9)$ & \\
\hline No & $52(86.7)$ & $96(88.9)$ & $98(98.0)$ & 0.015 & $96(71.1)$ & $140(87.0)$ & $128(92.1)$ & 0.000 \\
\hline \multicolumn{9}{|c|}{ Experience of nutrition counseling, $n(\%)$} \\
\hline Yes & $7(11.7)$ & $24(22.2)$ & $26(26.0)$ & 0.095 & $21(15.6)$ & $30(18.6)$ & $45(32.4)$ & 0.001 \\
\hline
\end{tabular}

$\mathrm{BMI}=$ body mass index; $\mathrm{ADL}=$ activities of daily living; NQ-E = nutritional quotient for elderly; SD, standard deviation; ${ }^{(1)}$ normal: $\leq 22.99 \mathrm{~kg} / \mathrm{m}^{2}$, overweight: $23.00-24.99 \mathrm{~kg} / \mathrm{m}^{2}$, obesity: $\geq 25.00 \mathrm{~kg} / \mathrm{m}^{2}$; ${ }^{(2)}$ multiple response; ${ }^{(3)}$ Etc.: bicycle, motorcycle, welfare center vehicle, elderly electric cart, and truck; ${ }^{(4)}$ social support: social care services for the elderly, home-delivered meal service, free meal service (elderly welfare facility, home support service center for the elderly, etc.); and $p$-values were determined using an independent $t$-test, one-way ANOVA test, scheffe's test (post hoc test), or chi-square test. 
Table 4. Living environmental factors of the subjects according to NQ-E level.

\begin{tabular}{|c|c|c|c|c|c|c|c|c|}
\hline & \multicolumn{8}{|c|}{ NQ-E levels $(n=703)$} \\
\hline & \multicolumn{3}{|c|}{ Men } & \multicolumn{5}{|c|}{ Women } \\
\hline & $\begin{array}{c}\text { Low } \\
(n=60)\end{array}$ & $\begin{array}{l}\text { Medium } \\
(n=108)\end{array}$ & $\begin{array}{c}\text { High } \\
(n=100)\end{array}$ & $p$-Value & $\begin{array}{c}\text { Low } \\
(n=135)\end{array}$ & $\begin{array}{l}\text { Medium } \\
(n=161)\end{array}$ & $\begin{array}{c}\text { High } \\
(n=139)\end{array}$ & $p$-Value \\
\hline \multicolumn{9}{|c|}{ Current living status, $n$ (\%) } \\
\hline Alone & $23(38.3)$ & $13(12.0)$ & $2(2.0)$ & & $89(65.9)$ & $61(37.9)$ & $28(20.1)$ & \\
\hline Living with spouse & $31(51.7)$ & $88(81.5)$ & $91(91.0)$ & 0.000 & $23(17.0)$ & $61(37.9)$ & $91(65.5)$ & 0.000 \\
\hline With children or children family & $6(10.0)$ & $7(6.5)$ & $7(7.0)$ & & $23(17.0)$ & $39(24.2)$ & $20(14.4)$ & \\
\hline \multicolumn{9}{|c|}{ Social relations, $n(\%)$} \\
\hline At least 3 times/week & $9(15.0)$ & $56(51.9)$ & $54(54.0)$ & & $47(34.8)$ & $73(45.3)$ & $93(66.9)$ & \\
\hline 1-2 times/week & $14(23.3)$ & $15(13.9)$ & $29(29.0)$ & & $23(17.0)$ & $35(21.7)$ & $27(19.4)$ & \\
\hline Less than 3 times/month & $17(28.3)$ & $24(22.2)$ & $16(16.0)$ & 0.000 & $27(20.0)$ & $38(23.6)$ & $18(12.9)$ & 0.000 \\
\hline Almost none & $20(33.3)$ & $13(12.0)$ & $1(1.0)$ & & $38(28.1)$ & $15(9.3)$ & $1(0.7)$ & \\
\hline \multicolumn{9}{|c|}{ Social activity ${ }^{(1)}, n(\%)$} \\
\hline Resting and hobby activities & $28(46.7)$ & 19 (17.6) & $11(11.0)$ & & $53(39.3)$ & $35(21.7)$ & $10(7.2)$ & \\
\hline Cultural and artistic activities & $5(8.3)$ & $6(5.6)$ & $12(12.0)$ & & $16(11.9)$ & $19(11.8)$ & $23(16.5)$ & \\
\hline Sports activities & $15(25.0)$ & $52(48.1)$ & $61(61.0)$ & 0.000 & $15(11.1)$ & $41(25.5)$ & $72(51.8)$ & 0.000 \\
\hline Other activities & $12(20.0)$ & $31(28.7)$ & $16(16.0)$ & & $51(37.8)$ & $66(41.0)$ & $34(24.5)$ & \\
\hline \multicolumn{9}{|c|}{ Frequency of social activity, $n(\%)$} \\
\hline Rarely & $34(56.7)$ & $26(24.1)$ & $5(5.0)$ & & $80(59.3)$ & $54(33.5)$ & $11(7.9)$ & \\
\hline Regular & $18(30.0)$ & $33(30.6)$ & $44(44.0)$ & 0.000 & $22(16.3)$ & $53(32.9)$ & $45(32.4)$ & 0.000 \\
\hline Trying to be active & $8(13.3)$ & $49(45.4)$ & $51(51.0)$ & & $33(24.4)$ & $54(33.5)$ & $83(59.7)$ & \\
\hline \multicolumn{9}{|c|}{ Food security, $n(\%)$} \\
\hline Food secure & $8(13.3)$ & $51(47.2)$ & $60(60.0)$ & & 29 (21.5) & $58(36.0)$ & $93(66.9)$ & \\
\hline Mildly food insecure & $38(63.3)$ & $48(44.4)$ & $38(38.0)$ & 0.000 & $76(56.3)$ & $83(51.6)$ & $41(29.5)$ & 0.000 \\
\hline Moderately/severely food insecure & $14(23.3)$ & $9(8.3)$ & $2(2.0)$ & & $30(22.2)$ & $20(12.4)$ & $5(3.6)$ & \\
\hline \multicolumn{9}{|c|}{ Ability to cook, $n(\%)$} \\
\hline Yes & $38(63.3)$ & $63(58.3)$ & $64(64.0)$ & 0.668 & $115(85.2)$ & 147 (91.3) & $133(95.7)$ & 0.010 \\
\hline No & $22(36.7)$ & $45(41.7)$ & $36(36.0)$ & & $20(14.8)$ & $14(8.7)$ & $6(4.3)$ & \\
\hline \multicolumn{9}{|c|}{ Nutrition knowledge ${ }^{(2)}, n(\%)$} \\
\hline Low & $29(48.3)$ & $17(15.7)$ & $15(15.0)$ & & $58(43.0)$ & $27(16.8)$ & $3(2.2)$ & \\
\hline Medium & $25(41.7)$ & $69(63.9)$ & $41(41.0)$ & 0.000 & $63(46.7)$ & $97(60.2)$ & $85(61.2)$ & 0.000 \\
\hline High & $6(10.0)$ & $22(20.4)$ & $44(44.0)$ & & $14(10.4)$ & $37(23.0)$ & $51(36.7)$ & \\
\hline Average nutrition knowledge, mean $\pm \mathrm{SD}$ & $3.57 \pm 3.01$ & $5.56 \pm 2.50$ & $6.34 \pm 2.70$ & 0.000 & $3.99 \pm 2.76$ & $5.85 \pm 2.71$ & $6.78 \pm 1.75$ & 0.000 \\
\hline
\end{tabular}

NQ-E, nutritional quotient for elderly; SD, standard deviation; ${ }^{(1)}$ resting and hobby activities: listening to music, reading/newspaper/magazine, napping, sauna, gardening, fishing, baduk/Korean chess (chang gi), cooking, etc.; cultural and artistic activities: movies, musical instruments, singing classes, arts, dancing, exhibition/museums, concerts, traditional art, etc.; sport activities: walking, badminton, ping-pong, swimming, running, billiards, etc.; other activities: religion, reunions, visiting relatives, speaking on the telephone, welfare facility, volunteer, etc.; (2) low: 0-3 score, medium: 4-7 score, and high: 8-10 score; and $p$-values were determined using an independent $t$-test, one-way ANOVA test, scheffe's test (post hoc test), or chi-square test. 
Table 5. Multinomial logistic regressions of an association between living environmental factors and NQ-E levels in elderly men.

\begin{tabular}{|c|c|c|c|c|c|c|}
\hline & \multicolumn{6}{|c|}{ Men $(n=268)$} \\
\hline & \multicolumn{3}{|c|}{ Medium-NQ-E } & \multicolumn{3}{|c|}{ High-NQ-E } \\
\hline & $\begin{array}{l}\text { Crude OR } \\
\text { (95\% CI) }\end{array}$ & $\begin{array}{c}\text { Model 1 } 1(1) \text { OR } \\
(95 \% \mathrm{CI})\end{array}$ & $\begin{array}{c}\text { Model 2(2) OR } \\
(95 \% \mathrm{CI})\end{array}$ & $\begin{array}{l}\text { Crude OR } \\
\text { (95\% CI) }\end{array}$ & $\begin{array}{l}\text { Model } 1 \text { OR } \\
\text { (95\% CI) }\end{array}$ & $\begin{array}{c}\text { Model } 2 \text { OR } \\
(95 \% \mathrm{CI})\end{array}$ \\
\hline \multicolumn{7}{|c|}{ Current living status, $n(\%)$} \\
\hline Alone & 1.00 & 1.00 & 1.00 & 1.00 & 1.00 & 1.00 \\
\hline Living with spouse & $5.02 * * *(2.27-11.10)$ & $3.10 *(1.21-7.93)$ & $2.74(0.97-7.75)$ & $33.75 * * *(7.52-151.48)$ & $11.75 * *(2.24-61.67)$ & $8.99 *(1.35-59.56)$ \\
\hline With children or children family & $2.06(0.57-7.46)$ & $0.68(0.14-3.31)$ & $0.40(0.07-2.22)$ & $13.41 * *(2.19-81.99)$ & $3.02(0.36-24.90)$ & $1.41(0.12-15.74)$ \\
\hline \multicolumn{7}{|c|}{ Social relations, $n(\%)$} \\
\hline At least 3 times/week & $9.57 * * *(3.55-25.80)$ & $2.60(0.71-9.50)$ & $1.75(0.41-7.43)$ & $120.00 * * *(14.27-1008.50)$ & $15.34 *(1.31-179.57)$ & $8.26(0.56-121.56)$ \\
\hline 1-2 times/week & $1.64(0.60-4.52)$ & $0.60(0.16-2.18)$ & $0.35(0.08-1.57)$ & $41.42 * *(5.03-340.76)$ & $8.69(0.75-99.91)$ & $4.42(0.29-65.60)$ \\
\hline Less than 3 times/month & $2.17(0.85-5.53)$ & $1.32(0.45-3.83)$ & $1.54(0.46-5.14)$ & $18.82 * *(2.25-157.01)$ & $7.19(0.63-82.00)$ & $8.78(0.60-127.48)$ \\
\hline Almost none & 1.00 & 1.00 & 1.00 & 1.00 & 1.00 & 1.00 \\
\hline \multicolumn{7}{|c|}{ Social activity ${ }^{(3)}, n(\%)$} \\
\hline Resting and hobby activities & 1.00 & 1.00 & 1.00 & 1.00 & 1.00 & 1.00 \\
\hline Cultural and artistic activities & $1.76(0.47-6.63)$ & $0.85(0.19-3.74)$ & $0.88(0.16-4.83)$ & $6.10^{* *}(1.74-21.42)$ & $1.69(0.36-7.78)$ & $2.56(0.43-15.16)$ \\
\hline Sports activities & $5.10^{* * * *}(2.25-11.57)$ & $2.89 *(1.10-7.53)$ & $3.41 *(1.18-9.80)$ & $10.35 * * *(4.21-25.39)$ & $2.41(0.74-7.78)$ & $2.86(0.75-10.79)$ \\
\hline Other activities & $3.80^{* *}(1.57-9.22)$ & $1.47(0.48-4.42)$ & $1.16(0.34-3.94)$ & $3.39 *(1.22-9.44)$ & $1.31(0.34-4.93)$ & $0.94(0.22-3.98)$ \\
\hline \multicolumn{7}{|c|}{ Frequency of social activity, $n(\%)$} \\
\hline Rarely & 1.00 & 1.00 & 1.00 & 1.00 & 1.00 & 1.00 \\
\hline Normal & $2.39 *(1.11-5.17)$ & $1.23(0.49-3.04)$ & $0.83(0.30-2.27)$ & $16.62 * * *(5.60-49.30)$ & $5.77^{* *}(1.61-20.73)$ & $2.56(0.65-10.12)$ \\
\hline Trying to be active & $8.01^{* * *}(3.24-19.80)$ & $2.85(0.96-8.43)$ & $2.26(0.70-7.29)$ & $43.35^{* * *}(13.07-143.73)$ & $9.04 * *(2.20-37.08)$ & $3.93(0.86-17.85)$ \\
\hline \multicolumn{7}{|c|}{ Food security, $n(\%)$} \\
\hline Food secure & $9.91 * * *(3.23-30.42)$ & $4.81 *(1.31-17.68)$ & $3.96(0.87-17.95)$ & $52.50 * * *(10.03-274.76)$ & $14.61^{* *}(2.08-102.16)$ & $6.57(0.73-58.98)$ \\
\hline Mildly food insecure & $1.96(0.76-5.02)$ & $1.29(0.43-3.82)$ & $0.71(0.18-2.73)$ & $7.00 *(1.48-32.92)$ & $3.22(0.51-20.06)$ & $1.02(0.12-8.29)$ \\
\hline Moderately/severely food insecure & 1.00 & 1.00 & 1.00 & 1.00 & 1.00 & 1.00 \\
\hline \multicolumn{7}{|c|}{ Nutrition knowledge ${ }^{(4)}, n(\%)$} \\
\hline Low & 1.00 & 1.00 & 1.00 & 1.00 & 1.00 & 1.00 \\
\hline Medium & $4.70 * * *(2.21-10.00)$ & $2.37(0.95-5.90)$ & $2.32(0.82-6.51)$ & $3.17^{* *}(1.42-7.03)$ & $0.74(0.24-2.26)$ & $0.49(0.13-1.82)$ \\
\hline High & $6.25 * *(2.11-18.47)$ & $1.95(0.49-7.70)$ & $1.71(0.35-8.31)$ & $14.17^{* * *}(4.92-40.77)$ & $1.16(0.27-4.92)$ & $0.76(0.13-4.21)$ \\
\hline
\end{tabular}

95\% CI, 95\% confidence interval; NQ-E, nutritional quotient for elderly; OR, odds ratio.; (1) model 1: adjusted for age, education, and monthly income; ${ }^{(2)}$ model 2: adjusted for age, education, monthly income, BMI, number of diseases, activities of daily living, experience of social support by government, food security, and nutrition knowledge; (3) resting and hobby activities: listening to music, reading/newspaper/magazine, napping, sauna, gardening, fishing, baduk/Korean chess (changgi)/yut, senior classes, cooking, etc.; cultural and artistic activities: movies, musical instruments, singing classes, art, dancing, exhibition/museums, concerts, traditional art, photography, etc.; sport activities: walking, badminton, ping-pong, swimming, running, billiards, etc.; other activities: religion, reunions, visiting relatives, speaking on the telephone, welfare facility, volunteer, etc.; ${ }^{(4)}$ low: $0-3$ score, medium: $4-7$ score, and high: 8-10 score; and $p$-values were determined using a multinomial logistic regressions analysis $\left({ }^{*} p<0.05,{ }^{* *} p<0.01\right.$, and $\left.{ }^{* * *} p<0.001\right)$. 
Table 6. Multinomial logistic regressions of an association between living environmental factors and NQ-E levels in elderly women.

\begin{tabular}{|c|c|c|c|c|c|c|}
\hline & \multicolumn{6}{|c|}{ Men $(n=268)$} \\
\hline & \multicolumn{3}{|c|}{ Medium-NQ-E } & \multicolumn{3}{|c|}{ High-NQ-E } \\
\hline & $\begin{array}{l}\text { Crude OR } \\
(95 \% \mathrm{CI})\end{array}$ & $\begin{array}{c}\text { Model } 1{ }^{(1)} \text { OR } \\
(95 \% \mathrm{CI})\end{array}$ & $\begin{array}{c}\text { Model 2 (2) OR } \\
(95 \% \mathrm{CI})\end{array}$ & $\begin{array}{c}\text { Crude OR } \\
(95 \% \mathrm{CI})\end{array}$ & $\begin{array}{c}\text { Model } 1 \text { OR } \\
(95 \% \mathrm{CI})\end{array}$ & $\begin{array}{c}\text { Model } 2 \text { OR } \\
(95 \% \mathrm{CI})\end{array}$ \\
\hline \multicolumn{7}{|c|}{ Current living status, $n(\%)$} \\
\hline Alone & 1.00 & 1.00 & 1.00 & 1.00 & 1.00 & 1.00 \\
\hline Living with spouse & $3.87 * * *(2.16-6.91)$ & $3.13^{* * *}(1.65-5.92)$ & $3.11 * *(1.52-6.35)$ & $12.57 * * *(6.73-23.47)$ & $5.44^{* * *}(2.61-11.33)$ & $5.62 * * *(2.36-13.38)$ \\
\hline With children or children family & $2.47 *(1.34-4.55)$ & $2.08 *(1.08-3.97)$ & $1.95(0.96-3.96)$ & $2.76^{* *}(1.32-5.76)$ & $1.70(0.73-3.93)$ & $1.88(0.72-4.88)$ \\
\hline \multicolumn{7}{|c|}{ Social relations, $n(\%)$} \\
\hline At least 3 times/week & $3.56 *(1.64-7.73)$ & $2.86^{* *}(1.35-6.08)$ & $2.35 *(1.02-5.40)$ & $25.33 * *(3.18-201.41)$ & $24.94 * *(3.10-200.17)$ & $16.04 *(1.72-149.18)$ \\
\hline $1-2$ times/week & $3.85 *(1.73-8.54)$ & $2.22(0.92-5.31)$ & $2.07(0.81-5.31)$ & $44.60 * * *(5.67-350.69)$ & $10.25 *(1.19-88.33)$ & $9.80(0.99-96.57)$ \\
\hline Less than 3 times/month & $3.93 * * *(1.95-7.93)$ & $2.88 *(1.28-6.47)$ & $2.70 *(1.12-6.51)$ & $75.19^{* * *}(10.01-564.74)$ & $11.03 *(1.28-94.59)$ & $9.42(0.94-93.74)$ \\
\hline Almost none & 1.00 & 1.00 & 1.00 & 1.00 & 1.00 & 1.00 \\
\hline \multicolumn{7}{|c|}{ Social activity ${ }^{(3)}, n(\%)$} \\
\hline Resting and hobby activities & 1.00 & 1.00 & 1.00 & 1.00 & 1.00 & 1.00 \\
\hline Cultural and artistic activities & $1.79(0.81-3.96)$ & $1.45(0.62-3.36)$ & $1.56(0.61-3.98)$ & $7.61^{* * *}(3.00-19.29)$ & $3.59 *(1.21-10.65)$ & $5.11 *(1.46-17.82)$ \\
\hline Sports activities & $4.13 * * *(1.99-8.58)$ & $3.40 * *(1.56-7.43)$ & $2.98 *(1.28-6.91)$ & $25.44 * * *(10.60-61.04)$ & $13.91 * * *(5.06-38.17)$ & $12.07 * * *(3.91-37.20)$ \\
\hline Other activities & $1.96 *(1.11-3.43)$ & $1.47(0.79-2.74)$ & $1.46(0.75-2.87)$ & $3.53 * *(1.58-7.88)$ & $2.34(0.92-5.91)$ & $2.32(0.81-6.58)$ \\
\hline \multicolumn{7}{|c|}{ Frequency of social activity, $n$ (\%) } \\
\hline Rarely & 1.00 & 1.00 & 1.00 & 1.00 & 1.00 & 1.00 \\
\hline Normal & $3.56^{* * *(1.94-6.53)}$ & $3.07 *(1.62-5.83)$ & $2.64 *(1.30-5.38)$ & $14.87^{* * *}(6.61-33.46)$ & $7.75 * * *(3.14-19.11)$ & $5.75 *(2.09-15.76)$ \\
\hline Trying to be active & $2.42 *(1.39-4.21)$ & $1.95 *(1.07-3.53)$ & $1.41(0.72-2.75)$ & $18.29^{* * * *}(8.65-38.65)$ & $9.45 * * *(4.11-21.71)$ & $5.28 * *(2.05-13.58)$ \\
\hline \multicolumn{7}{|c|}{ Food security, $n$ (\%) } \\
\hline Food secure & $3.00 * *(1.46-6.16)$ & $2.81 *(1.29-6.13)$ & $1.79(0.73-4.41)$ & $19.241^{* * *}(6.83-54.137)$ & $10.79 * * *(3.44-33.86)$ & $5.07 *(1.35-18.97)$ \\
\hline Mildly food insecure & $1.63(0.85-3.12)$ & $1.52(0.76-3.04)$ & $1.40(0.64-3.06)$ & $3.23 *(1.16-8.97)$ & $2.28(0.74-7.01)$ & $1.98(0.54-7.17)$ \\
\hline Moderately/severely food insecure & 1.00 & 1.00 & 1.00 & 1.00 & 1.00 & 1.00 \\
\hline \multicolumn{7}{|c|}{ Nutrition knowledge $^{(4)}, n(\%)$} \\
\hline Low & 1.00 & 1.00 & 1.00 & 1.00 & 1.00 & 1.00 \\
\hline Medium & $3.30 * * *(1.89-5.76)$ & $2.87 * *(1.58-5.20)$ & $2.61^{* *}(1.36-5.00)$ & $26.08 * * *(7.81-87.06)$ & $19.86^{* * *}(5.17-76.15)$ & $17.71^{* * *}(4.14-75.763)$ \\
\hline High & $5.67^{* * *}(2.63-12.21)$ & $3.52 * *(1.52-8.17)$ & $3.50 * *(1.35-9.03)$ & $70.42 * * *(19.14-259.06)$ & $23.61 * * *(5.46-102.12)$ & $20.49 * * *(4.12-101.73)$ \\
\hline
\end{tabular}

95\% CI, 95\% confidence interval; NQ-E, nutritional quotient for elderly; OR, odds ratio; ${ }^{(1)}$ model 1: adjusted for age, education, and monthly income; ${ }^{(2)}$ model 2: adjusted for age, education, monthly income, BMI, number of diseases, activities of daily living, experience of social support by government, food security, and nutrition knowledge; (3) resting and hobby activities: listening to music, reading/newspaper/magazine, napping, sauna, gardening, fishing, baduk/Korean chess (changgi)/yut, senior classes, cooking, etc.; cultural and artistic activities: movies, musical instruments, singing classes, art, dancing, exhibition/museums, concerts, traditional art, photography, etc.; sport activities: walking, badminton, ping-pong, swimming, running, billiards, etc.; other activities: religion, reunions, visiting relatives, speaking on the telephone, welfare facility, volunteer, etc.; ${ }^{(4)}$ low: $0-3$ score, medium: $4-7$ score, and high: 8-10 score; and $p$-values were determined using a multinomial logistic regressions analysis $\left({ }^{*} p<0.05,{ }^{* *} p<0.01\right.$, and $\left.{ }^{* * *} p<0.001\right)$. 


\section{Discussion}

This study examined the association between NQ-E and various elements of one's living environment including health-related factors, food- and nutrition-related factors, social activity, and mobility factors by sex. Social activity and networks were important to good nutritional status and a healthy life in all participants. The results of NQ-E were closely related to one's living environment. NQ-E in both men and women was particularly closely associated with living status and social activities. Furthermore, the nutrition quotient of elderly women was additionally related to various living environmental factors such as social relations, food security, and nutrition knowledge. We suggest that it may be helpful to consider sex differences in dietary management for elderly people.

Based on the NQ-E results among the male participants, high scores on dietary behavior and diversity are thought to be related to social support. Social support of various types is associated with positive health outcomes [24]. Importantly, whether one is living alone or not, the main factor for evaluating social support in elderly people is malnutrition $[7,25]$. Not surprisingly, living with a spouse was an important factor in social and emotional stability. Older adults' closest emotional connections are with their spouses or partners $[7,14]$. Moreover, life satisfaction is also high among elderly people who live a spouse-oriented life $[7,14,25]$. Older people living with their spouses usually have well-managed health care and better nutritional status than those who live alone [26-28]. In this study, the rate of living with a spouse was twice as high in men as in women. It is thought that this difference played a role in men's diverse food intake and high NQ-E score. This result suggests that older people who live alone may need social care services that provide social and emotional support, both for nutrition management and good nutritional status and for overall health.

In this study, women's low NQ-E scores were primarily due to low scores for dietary behavior, which includes questions about difficulties in chewing foods, perception of one's health, depressive symptoms, handwashing practices before eating meals, hours of exercise, and efforts to have healthy eating habits. Among the participants in this study, $40.9 \%$ of elderly women was living alone, which was higher than the $27.4 \%$ reported in another study $[29,30]$. Thus, it may be that women reported more depressive feelings because of the length of time they lived alone, which tends to be longer for women than for men due to differences in life expectancy. In addition, women's job levels and income are often lower than those of men, which may contribute to depression among elderly women. Therefore, it is thought that these components of dietary behavior among the women in this study may partially explain the lower NQ-E scores. Because exercise reduces depression, sports activities are often recommended to relieve its effects [2,31]. However, elderly women are also known to have more difficulty participating in exercise than men.

In both elderly men and women, NQ-E was positively associated with living with one's spouse and with engaging in a fair amount of social activity. In women, however, social relations, food security, and nutrition knowledge were additionally related to NQ-E, suggesting that various elements of the living environment are more closely linked to nutrition in women than in men. For these reasons, we need to focus on increasing older people's social networks. Recently, the importance of social networks for a healthy life has emerged in addition to physical health [24,31]. However, in reality, elderly people tend to have a limited social network after a spouse's death, which may be compounded by economic problems and retirement from work $[7,14]$. Social networks have also been reported to affect depression in elderly people, a major factor influencing food intake [7]. Therefore, social networks are very important for improving nutritional status among elderly people as well as their social health and quality of life. Kim et al.'s findings support the conclusion that the better the social network is, the lower the nutritional risk will be [14,24].

In the present study, elderly men living with a spouse had higher nutrition quotients than did women with the same living status. This is likely due to the large influence of a spouse on men's social networks. Men who live alone reported having poorer meal quality and lower food intake than men who lived with a spouse [32]. In addition, men who lived alone had high intake of convenience foods 
and high-fat/high-cholesterol meals [33]. This means that living with a spouse is a very important factor in food intake and nutritional status.

Women with higher NQ-Es had the highest rates of living with a spouse and of engaging in social activities. In other words, women with more frequent social relations and activities and with greater food security and nutritional knowledge had higher NQ-E scores than women with lower values in those areas. Thus, elements of the living environment were more closely linked to women's NQ-E levels than to that of men. Therefore, a multicomponent approach is needed to improve the nutritional status and health of elderly women. These results suggest that the social health and nutritional status of both men and women will be improved by encouraging social activities, such as sports activities, that can create a social network. In addition, a combined approach that addresses multiple factors, such as nutrition education and social activities, may be needed to improve elderly women's nutritional status and health.

When women's nutritional knowledge score was medium or high, their nutritional status was significantly better, suggesting that being health conscious may improve nutritional status. Women were most interested in the topics of health and happiness and they participated in more educational programs, social services, and cultural activities than did men [34]. Concern about health and nutritional knowledge in women reportedly influences desirable eating habits [35]. Women's high level of interest in health may be related to their chronic diseases. This suggestion is in line with Timpini et al. [36], who found that women had a higher prevalence of disease than men. In the present study, the average number of diseases reported by elderly women with low NQ-E scores was higher than that in elderly men with similar NQ-E scores. Thus, continuous nutrition education and management support for women are likely to greatly improve their health. These findings suggest that chronic disease-related nutrition education will be very useful in enhancing dietary behavior and nutritional status.

Some limitations of this study should be noted. First, the cross-sectional nature of the study limits our ability to invoke causal relationships between the nutrition quotient and elements of the participants' living environments. Second, since the study was conducted as a self-administered questionnaire, we had to exclude the elderly with poor literacy. This may have excluded some older people who may be more nutritionally vulnerable. Third, the uncertainty level is high for some factors, as indicated by the wide confidence intervals seen in the results of the multinomial logistic regression analyses. This seems to have resulted from the low numbers of participants in some categories; hence, longitudinal studies of the relevant factors are necessary. Meanwhile, the main strength of this study is that the results were obtained using data collected through large-scale nationwide surveys of elderly people in various regions of the country. It is also meaningful that the study analyzed various factors of the living environment according to sex, which can clearly affect nutritional status in elderly community-dwelling people.

\section{Conclusions}

We examined the associations between food quality, food behavior, and nutritional status and living environmental factors in Koreans over 65 years old using the NQ-E. NQ-E scores, including dietary behavior and diversity, of elderly men were significantly higher than those of elderly women. The living situations and social activities of both men and women were related to nutritional status. In particular, elderly individuals living with a spouse and those who played sports had higher nutrition quotients than those who lived alone and those with sedentary lives. Meanwhile, women who experienced food security and had high levels of nutrition knowledge showed higher nutrition quotients than those experiencing moderate or severe food insecurity and low nutrition knowledge. Our results confirm the existence of sex differences in the relationship between the nutrition quotient and living environmental factors such as social activity and support systems. It is important to educate elderly individuals about the role of social networks and relationships in improving their nutritional status. Elderly people should be provided with opportunities for social activities to improve their nutritional status. 
Author Contributions: Conceptualization and study design, D.E.K., H.-S.L., Y.S.K. and Y.K.P.; methodology, D.E.K. and Y.K.P.; investigation, D.E.K. and H.-S.L.; data curation, D.E.K., H.-S.L., and H.A.; writing一original draft preparation D.E.K., H.-S.L. and H.A.; writing-review and editing, Y.S.K., H.A. and Y.K.P.; supervision, Y.S.K. and Y.K.P. All authors have read and agreed to the published version of the manuscript.

Funding: This work was supported by a research grant from the Korea Health Technology R \& D Project through the Korea Health Industry Development Institute (KHIDI), which is funded by the Ministry of Health \& Welfare, Republic of Korea (grant number: HI18C0479).

Conflicts of Interest: The authors declare no conflict of interest.

\section{References}

1. Koohsari, M.J.; Nakaya, T.; Oka, K. Activity-friendly built environments in a super-aged society, japan: Current challenges and toward a research agenda. Int. J. Environ. Res. Public Health 2018, 15, 2054. [CrossRef] [PubMed]

2. Statistics. World Population Prospects: The 2019 Revision. Available online: http://kostat.go.kr/portal/korea/ kor_nw/1/1/index.board?bmode=read\&aSeq=377226 (accessed on 1 June 2020).

3. Plouffe, L.; Kalache, A. Towards global age friendly cities: Determining urban features that promote active aging. J. Urban Health 2010, 87, 733-739. [CrossRef] [PubMed]

4. Rogers, N.T.; Marshall, A.; Roberts, C.H.; Demakakos, P.; Steptoe, A.; Scholes, S. Physical activity and trajectories of frailty among older adults: Evidence from the English Longitudinal Study of Ageing. PLoS ONE 2017, 12, e0170878. [CrossRef] [PubMed]

5. Kahan, S.; Kushner, R.F. Practice-Based Nutrition Care. In An Issue of Medical Clinics of North America, 1st ed; Elsevier: Philadelphia, PA, USA, 2016; pp. 28-49.

6. Galenkamp, H.; Deeg, D.J. Increasing social participation of older people: Are there different barriers for those in poor health? Introduction to the special Section. Eur. J. Ageing 2016, 13, 87-90.

7. Brownie, S. Why are elderly individuals at risk of nutritional deficiency? Int. J. Nurs. Pract. 2006, 12, 110-118. [CrossRef]

8. Torres, M.J.; Dorigny, B.; Kuhn, M.; Berr, C.; Barberger-Gateau, P.; Letenneur, L. Nutritional status in community-dwelling elderly in france in urban and rural areas. PLoS ONE 2014, 9, e10513. [CrossRef]

9. Leslie, W.; Hankey, C. Aging, Nutritional Status and Health. Healthcare 2015, 3, 648-658. [CrossRef]

10. Poda, G.G.; Hsu, C.Y.; Rau, H.H.; Chao, J.C.J. Impact of socio-demographic factors, lifestyle and health status on nutritional status among the elderly in Taiwan. Nutr. Res. Pract. 2019, 13, 222-229. [CrossRef]

11. Kiefer, I.; Rathmanner, T.; Kunze, M. Eating and dieting differences in men and women. J. Men's Health Gend. 2005, 2, 194-201. [CrossRef]

12. Li, C. Gender differences in nutrition knowledge, attitude, and practice among elderly people. Int. J. Manag. Econ. Soc. Sci. (IJMESS) 2017, 6, 199-211.

13. Rurik, I. Nutritional differences between elderly men and women. primary care evaluation in hungary. Ann. Nutr. Metab. 2006, 50, 45-50. [CrossRef] [PubMed]

14. Drewnowski, A.; Evans, W.J. Nutrition, physical activity, and quality of life in older adults: Summary. J. Gerontol. Ser. A Biol. Sci. Med Sci. 2001, 56, 89-94. [CrossRef] [PubMed]

15. Lim, H.S. Association of Dietary Variety Status and Sarcopenia in Korean Elderly. J. Bone Metab. 2020, 27, 143-149. [CrossRef] [PubMed]

16. Lim, H.S.; Lee, M.N. Comparison of Health Status and Nutrient Intake by Household Type in the Elderly Population. J. Bone Metab. 2019, 26, 25-30. [CrossRef] [PubMed]

17. Su, Y.; Hirayama, K.; Han, T.; Izutsu, M.; Yuki, M. Sarcopenia Prevalence and Risk Factors among Japanese Community Dwelling Older Adults Living in a Snow-Covered City According to EWGSOP2. J. Clin. Med. 2019, 8, 291. [CrossRef] [PubMed]

18. Israel, G.D. Determining Sample Size. University of Florida Cooperative Extension Service, Institute of Food and Agriculture Sciences, EDIS, Florida. 1992. Available online: http:/www.gjimt.ac.in/web/wp-content/ uploads/2017/10/2_Glenn-D.-Israel_Determining-Sample-Size.pdf (accessed on 25 May 2020).

19. Sudman, S. Applied sampling. In Handbook of Survey Research; Academic Press: New York, NY, USA, 1976; Volume 6, pp. 145-230. 
20. Chung, M.; Kwak, T.; Kim, H.; Kang, M.; Lee, J.; Chung, H.R.; Kwon, S.; Hwang, J.; Choi, Y. Development of NQ-E, nutrition quotient for korean elderly: Item selection and validation of factor structure. J. Nutr. Health 2018, 51, 87-102. [CrossRef]

21. Lim, J.U.; Lee, J.H.; Kim, J.S.; Hwang, Y.I.; Kim, T.H.; Lim, S.Y.; Yoo, K.H.; Jung, K.S.; Kim, Y.K.; Rhee, C.K. Comparison of world health organization and asia-pacific body mass index classifications in COPD patients. Int. J. Chron. Obstruct. Pulm. Dis. 2017, 12, 2465-2475. [CrossRef]

22. Lee, S.; Lee, K.W.; Oh, J.E.; Cho, M.S. Nutritional and health consequences are associated with food insecurity among Korean elderly: Based on the fifth (2010) Korea national health and nutrition examination survey (KNHANES V-1). J. Nutr. Health 2015, 48, 519-529. [CrossRef]

23. Shin, S.; Hwang, E. Gender differences in the cognitive function and nutritional status in older age: A representative nationwide data of Korean elders. J. Korean Public Health Nurs. 2017, 31, $209-219$.

24. Aroogh, M.D.; Shahboulaghi, F.M. Social participation of older adults: A concept analysis. Int. J. Community Based Nurs. Midwifery 2020, 8, 55-72.

25. Robinson, S.M. Improving nutrition to support healthy ageing: What are the opportunities for intervention? Proc. Nutr. Soc. 2018, 77, 257-264. [CrossRef] [PubMed]

26. Charlton, K.E. Elderly men living alone: Are they at high nutritional risk? J. Nutr. Health Aging 1999, 3 , 42-47. [PubMed]

27. De Morais, C.; Oliveira, B.; Afonso, C.; Lumbers, M.; Raats, M.; De Almeida, M. Nutritional risk of european elderly. Eur. J. Clin. Nutr. 2013, 67, 1215-1219. [CrossRef] [PubMed]

28. Damião, R.; Santos, Á.D.S.; Matijasevich, A.; Menezes, P.R. Factors associated with risk of malnutrition in the elderly in south-eastern brazil. Rev. Bras. Epidemiol. 2017, 20, 598-610. [CrossRef]

29. Gham, M.; Um, M.; Kye, S. Evaluation of dietary quality and nutritional status based on nutrition quotient and health functional food intake in the Korea elderly. J. Korean Soc. Food Cult. 2019, 34, 474-485.

30. Choi, K.; Jeon, G.; Jang, K. Gender differences in the impact of intergenerational support on depressive symptoms among older adults in korea. Int. J. Environ. Res. Public Health 2020, 17, 4380. [CrossRef]

31. Rapacciuolo, A.; Perrone Filardi, P.; Cuomo, R.; Mauriello, V.; Quarto, M.; Kisslinger, A.; Savarese, G.; Illario, M.; Tramontano, D. The impact of social and cultural engagement and dieting on well-being and resilience in a group of residents in the metropolitan area of naples. J. Aging Res. 2016, 4768420. [CrossRef]

32. Nam, E.; Lee, J. Effects of household type on blood pressure, body mass index, mini nutritional assessment score, and biochemical indicators in elderly individuals living alone and with families. J. Korean Acad. Soc. Home Care Nurs. 2019, 26, 210-218.

33. Park, J.K.; Son, S.M. The dietary behaviors, depression rates and nutrient intakes of the elderly females living alone. Korean J. Community Nutr. 2003, 8, 716.

34. Kim, H.; Lee, K. The relationship between the participation of the female elderly in the leisure activity, and physical health and a sense of happiness. J. Sports Leis. Stud. 2008, 34, 671-680.

35. Yim, K.S.; Min, Y.H.; Lee, T.Y.; Kim, Y.J. Strategies to improve nutrition for the elderly in suwon: Analysis of dietary behavior and food preferences. Korean J. Community Nutr. 1998, 3, 410-422.

36. Timpini, A.; Facchi, E.; Cossi, S.; Ghisla, M.; Romanelli, G.; Marengoni, A. Self-reported socio-economic status, social, physical and leisure activities and risk for malnutrition in late life: A cross-sectional population-based study. J. Nutr. Health Aging 2011, 15, 233-238. [CrossRef] [PubMed]

(C) 2020 by the authors. Licensee MDPI, Basel, Switzerland. This article is an open access article distributed under the terms and conditions of the Creative Commons Attribution (CC BY) license (http://creativecommons.org/licenses/by/4.0/). 